\title{
An alternative quadratic functional equation on 2-divisible groups
}

\author{
Paisan Nakmahachalasint \\ Department of Mathematics and Computer Science, Faculty of Science, Chulalongkorn University, \\ Bangkok 10330 Thailand
}

e-mail: Paisan.N@chula.ac.th

Received 10 Nov 2014

Accepted 29 Jan 2015

ABSTRACT: Let $(G, \cdot)$ be a 2-divisible group and let $\left(G^{*},+\right)$ be a uniquely divisible abelian group. We show that the alternative quadratic functional equation $f\left(x y^{-1}\right)+f(x y)= \pm 2(f(x)+f(y))$ is equivalent to the quadratic functional equation $f\left(x y^{-1}\right)+f(x y)=2 f(x)+2 f(y)$ for the class of functions $f: G \rightarrow G^{*}$.

KEYWORDS: alternative functional equation, polynomial-type functional equation, bi-additive function

MSC2010: 39B52 39B05

\section{INTRODUCTION}

The well-known alternative Cauchy functional equation

$$
\|f(x+y)\|=\|f(x)+f(y)\|
$$

has been studied by several authors ${ }^{1,2}$. A general alternative form,

$$
\begin{aligned}
\{c f(x+y)- & a f(x)-b f(y)-d\} \\
& \times\{f(x+y)-f(x)-f(y)\}=0,
\end{aligned}
$$

has been solved by Forti ${ }^{3}$ by extending the work of Ger $^{4}$ as well as that of Forti and Paganoni ${ }^{5,6}$. Readers who are interested in recent work on alternative Cauchy functional equations, should refer to, e.g., Refs. 7, 8 and references therein.

Skof ${ }^{9}$ took the classical quadratic functional equation

$$
f(x+y)+f(x-y)=2 f(x)+2 f(y)
$$

and derived the following four alternative forms of (1):

$$
\begin{aligned}
|f(x+y)| & =|2 f(x)+2 f(y)-f(x-y)|, \\
|f(x-y)| & =|2 f(x)+2 f(y)-f(x+y)|, \\
|2 f(y)| & =|f(x+y)+f(x-y)-2 f(x)|, \\
|2 f(x)| & =|f(x+y)+f(x-y)-2 f(y)| .
\end{aligned}
$$

Skof proved that for the class of functions $f: X \rightarrow \mathbb{R}$, where $X$ is a real linear space, each of the above functional equations is equivalent to (1). Nevertheless, there remains another alternative form of (1), that is

$$
|f(x+y)+f(x-y)|=|2 f(x)+2 f(y)|,
$$

for which Skof only proved that $f$ is rationally homogeneous of degree 2, i.e., $f(r x)=r^{2} f(x)$ for all rational numbers $r$ and for all $x \in X$. However, the equivalence of (1) and (2) can be established for the case when $X=\mathbb{R}$ and $f$ is a continuous function.

Let $(G, \cdot)$ be a 2-divisible group, i.e., for every $x \in G$, there exists $y \in G$ such that $y^{2}=x$. Let $\left(G^{*},+\right)$ be a uniquely divisible abelian group, i.e., for every positive integer $n$ and every $x \in G$, there exists a unique $y \in G$ such that $n y=x$. We will complete and generalize the remaining work of Skof by showing that the alternative quadratic functional equation

$$
f\left(x y^{-1}\right)+f(x y)= \pm 2(f(x)+2 f(y))
$$

is equivalent to the quadratic functional equation

$$
f\left(x y^{-1}\right)+f(x y)=2 f(x)+2 f(y)
$$

for the more general class of functions $f: G \rightarrow G^{*}$ with no additional condition.

\section{SOME BASIC RESULTS}

In the proofs presented in this paper, there will be many substitutions into the alternative quadratic functional equation (3). Therefore it is convenient to denote the statement

$\mathscr{P}_{f}(x, y):=\left(f\left(x y^{-1}\right)+f(x y)= \pm 2(f(x)+f(y))\right)$ 
for any function $f: G \rightarrow G^{*}$. The set of all solutions of (3) will be denoted by

$\mathscr{A}\left(G, G^{*}\right):=\left\{f: G \rightarrow G^{*} \mid \mathscr{P}_{f}(x, y)\right.$ for all $\left.x, y \in G\right\}$.

In this section, we aim to prove a basic theorem that embodies the quadratic nature of the alternative quadratic functional equation (3) on groups. We will begin by first proving some fundamental lemmas that will finally lead to the proof of that basic theorem. It should be noted that the 2divisibility of $G$ will not be used in this section.

Lemma 1 Let $e$ be the identity of $(G, \cdot)$. If $f \in$ $\mathscr{A}\left(G, G^{*}\right)$, then $f(e)=0$.

Proof: Let $f \in \mathscr{A}\left(G, G^{*}\right)$. Considering $\mathscr{P}_{f}(e, e)$, we conclude that $f(e)=0$.

Lemma 2 If $f \in \mathscr{A}\left(G, G^{*}\right)$, then $f\left(x^{2}\right)=4 f(x)$ for every $x \in G$.

Proof: Let $f \in \mathscr{A}\left(G, G^{*}\right)$. Considering $\mathscr{P}_{f}(x, x)$ and using Lemma 1 , we have

$$
f\left(x^{2}\right)= \pm 4 f(x)
$$

for every $x \in G$. Suppose there exist $a \in G$ such that

$$
f\left(a^{2}\right) \neq 4 f(a) .
$$

Setting $x=a$ in (5) and noting (6), we have

$$
f\left(a^{2}\right)=-4 f(a) .
$$

From (6) and (7), we observe that

$$
f(a) \neq 0 .
$$

Setting $x=a^{2}$ in (5) and using (7), we have

$$
f\left(a^{4}\right)= \pm 16 f(a) .
$$

Considering $\mathscr{P}_{f}\left(a^{2}, a\right)$ and using (7), we have

$$
f\left(a^{3}\right) \in\{5 f(a),-7 f(a)\} .
$$

Considering $\mathscr{P}_{f}\left(a^{3}, a\right)$, then using (7) and (10), we have

$$
f\left(a^{4}\right) \in\{16 f(a),-8 f(a)\} .
$$

Comparing (9) and (11), while keeping (8) in mind, we infer that

$$
f\left(a^{4}\right)=16 f(a) .
$$

Consider $\mathscr{P}_{f}\left(a^{3}, a^{2}\right)$, then using (7) and (10), we have

$$
f\left(a^{5}\right) \in\{f(a),-3 f(a), 21 f(a),-23 f(a)\} .
$$

Consider $\mathscr{P}_{f}\left(a^{4}, a\right)$, then using (10), and (12), we have

$$
f\left(a^{5}\right) \in\{29 f(a), 41 f(a),-39 f(a),-27 f(a)\} .
$$

Comparing (13) and (14), we must have $f(a)=0$, which contradicts (8). Hence we have the desired result.

Corollary 1 If $f \in \mathscr{A}\left(G, G^{*}\right)$, then $f\left(x^{4}\right)=16 f(x)$ for every $x \in G$.

Proof: Apply Lemma 2 twice.

Lemma 3 If $f \in \mathscr{A}\left(G, G^{*}\right)$, then $f\left(x^{3}\right)=9 f(x)$ for every $x \in G$.

Proof: Let $f \in \mathscr{A}\left(G, G^{*}\right)$. Suppose there exists $a \in G$ such that

$$
f\left(a^{3}\right) \neq 9 f(a) .
$$

From Lemma 2, we have $f\left(a^{2}\right)=4 f(a)$. Considering $\mathscr{P}_{f}\left(a^{2}, a\right)$ and (15), we have

$$
f\left(a^{3}\right)=-11 f(a) .
$$

Considering $\mathscr{P}_{f}\left(a^{3}, a^{2}\right)$ and (16), we have

$$
f\left(a^{5}\right) \in\{13 f(a),-15 f(a)\} .
$$

Considering $\mathscr{P}_{f}\left(a^{4}, a\right)$, then using (16) and $f\left(a^{4}\right)=$ $16 f(a)$ from Corollary 1 , we have

$$
f\left(a^{5}\right) \in\{45 f(a),-23 f(a)\} .
$$

Comparing (17) and (18), we conclude that $f(a)=$ 0 . Then (16) gives $f\left(a^{3}\right)=0$. So we have $f\left(a^{3}\right)=$ $9 f(a)=0$, which contradicts (15).

Lemma 4 If $f \in \mathscr{A}\left(G, G^{*}\right)$, then $f\left(x^{-1}\right)=f(x)$ for every $x \in G$.

Proof: Let $f \in \mathscr{A}\left(G, G^{*}\right)$ and let $e$ be the identity of $(G, \cdot)$. Considering $\mathscr{P}_{f}(e, x)$ and $f(e)=0$, we get

$$
f\left(x^{-1}\right) \in\{f(x),-3 f(x)\}
$$

for every $x \in G$. Suppose there exists $a \in G$ such that

$$
f\left(a^{-1}\right) \neq f(a) .
$$

Setting $x=a$ in (19) and noting (20), we have $f\left(a^{-1}\right)=-3 f(a)$. Setting $x=a^{-1}$ in (19) and noting (20) again, we have $f(a)=-3 f\left(a^{-1}\right)$. Thus $f\left(a^{-1}\right)=f(a)=0$, which contradicts (20).

Having all the lemmas, we are now ready to prove the following theorem by induction. 
Theorem 1 If $f \in \mathscr{A}\left(G, G^{*}\right)$, then $f\left(x^{n}\right)=n^{2} f(x)$ for every $x \in G, n \in \mathbb{Z}$.

Proof: Let $f \in \mathscr{A}\left(G, G^{*}\right)$ and let $x \in G$. Let $P(n)$ denote the statement $f\left(x^{n}\right)=n^{2} f(x)$. From Lemma 2, Lemma 3 and Corollary 1, we can see that $P(n)$ already holds for $n=1,2,3,4$. Let $k \geqslant$ 4 be an integer, and suppose that $P(n)$ holds for $n=1,2, \ldots, k$. Suppose that $P(n)$ does not hold for $n=k+1$, that is

$$
f\left(x^{k+1}\right) \neq(k+1)^{2} f(x) .
$$

Considering $\mathscr{P}_{f}\left(x^{k}, x\right)$, then using $P(k-1), P(k)$ and (21), we have

$$
f\left(x^{k+1}\right)=\left(-3 k^{2}+2 k-3\right) f(x) .
$$

Considering $\mathscr{P}_{f}\left(x^{k-1}, x^{2}\right)$, then using $P(k-3), P(k-$ $1), P(2)$ and (21), we have

$$
f\left(x^{k+1}\right)=\left(-3 k^{2}+10 k-19\right) f(x) .
$$

From (22), (23) and that $k \geqslant 4$, we conclude that $f(x)=0$. Now (23) gives $f\left(x^{k+1}\right)=0$. So $f\left(x^{k+1}\right)=(k+1)^{2} f(x)=0$, which contradicts (21). Hence $P(n)$ holds for $n=k+1$.

By induction, $P(n)$ now holds for every $n \in \mathbb{N}$ where $\mathbb{N}$ is the set of positive integers. That is $f\left(x^{n}\right)=n^{2} f(x)$ for every $x \in G, n \in \mathbb{N}$. For $f\left(x^{n}\right)$ with $n \leqslant 0$, we can apply Lemma 1 and Lemma 4 to complete the proof.

The following theorem shows that for a finite group $(G, \cdot)$, the only function satisfying the quadratic functional equation (3) is the zero function.

Theorem 2 If $(G, \cdot)$ is a finite group, then $f \in$ $\mathscr{A}\left(G, G^{*}\right)$ if and only if $f(x)=0$ for every $x \in G$.

Proof: If $(G, \cdot)$ is a finite group with the identity $e$, then, for every $x \in G$, there exists $n \in \mathbb{N}$ such that $x^{n}=e$. Therefore $f\left(x^{n}\right)=f(e)$. From Theorem 1, we know that $f\left(x^{n}\right)=n^{2} f(x)$ and $f(e)=0$. Hence $f(x)=0$.

For an infinite cyclic group $G=\langle a\rangle$, Theorem 1 yields $f\left(a^{n}\right)=n^{2} f(a)$ for every $n \in \mathbb{Z}$, which suffices to define the general solution. The next theorem demonstrates that for the additive group on rational numbers, we can still apply Theorem 1 to determine the general solution.

Theorem 3 A function $f: \mathbb{Q} \rightarrow \mathbb{R}$ satisfies

$$
f(x-y)+f(x+y)= \pm 2(f(x)+f(y))
$$

for every $x, y \in \mathbb{Q}$ if and only if there exists a real constant $k$ such that $f(x)=k x^{2}$ for every $x \in \mathbb{Q}$.
Proof: By Theorem 1, we have $f(n x)=n^{2} f(x)$ for every $x \in \mathbb{Q}, n \in \mathbb{Z}$. For a rational number $x=p / q$ with $p \in \mathbb{Z}$ and $q \in \mathbb{Z} \backslash\{0\}$,

$$
\begin{aligned}
f(x) & =f\left(\frac{p}{q}\right)=p^{2} f\left(\frac{1}{q}\right)=\frac{p^{2}}{q^{2}} \cdot q^{2} f\left(\frac{1}{q}\right) \\
& =\frac{p^{2}}{q^{2}} f(1)=k x^{2},
\end{aligned}
$$

where $k=f(1)$.

The sufficiency of the theorem is obvious.

\section{AUXILIARY THEOREM AND LEMMAS}

This section provides some lemmas and a theorem that will be required in the main results. Let us start with the following lemma which states a necessary condition for function values taken at four consecutive points in a sequence.

Lemma 5 Let $f \in \mathscr{A}\left(G, G^{*}\right)$. For every $x, y \in G$, there exist $\varepsilon_{1}, \varepsilon_{2} \in\{-1,1\}$ such that

$f\left(x y^{-1}\right)+f\left(x y^{2}\right)=\varepsilon_{1}(f(x)+f(x y))+4 \varepsilon_{2} f(y)$.

Proof: Let $f \in \mathscr{A}\left(G, G^{*}\right)$ and let $x, y \in G$. Since $(G, \cdot)$ is 2-divisible, there exists $z \in G$ such that $y=$ $z^{2}$.

Eliminating $f(x z)$ from $\mathscr{P}_{f}(x z, z)$ and $\mathscr{P}_{f}\left(x z, z^{3}\right)$, then using $f\left(z^{3}\right)=9 f(z)$, we get the desired result.

The following three lemmas give some necessary conditions when some alternatives in $\mathscr{P}_{f}(x, y)$ and $\mathscr{P}_{f}(x y, y)$ are decided.

Lemma 6 Let $f \in \mathscr{A}\left(G, G^{*}\right)$ and let $x, y \in G$. If

$$
\begin{aligned}
f\left(x y^{-1}\right)+f(x y) & =-2 f(x)-2 f(y), \\
f(x)+f\left(x y^{2}\right) & =-2 f(x y)-2 f(y),
\end{aligned}
$$

then $f(x)+f(x y) \in\{0,-2 f(y),-4 f(y)\}$.

Proof: Assuming the hypotheses in the lemma, and adding up the two equations therein, we have

$$
\left(f\left(x y^{-1}\right)+f\left(x y^{2}\right)\right)+3(f(x)+f(x y))=-4 f(y),
$$

which yields the desired result when combined with Lemma 5.

Lemma 7 Let $f \in \mathscr{A}\left(G, G^{*}\right)$ and let $x, y \in G$. If

$$
\begin{aligned}
f\left(x y^{-1}\right)+f(x y) & =-2 f(x)-2 f(y), \\
f(x)+f\left(x y^{2}\right) & =2 f(x y)+2 f(y),
\end{aligned}
$$

then $f(x)= \pm f(y)$ or $f(x y)=f(x) \pm 2 f(y)$. 
Proof: Assuming the hypotheses in the lemma, and adding up the two equations therein, we have

$$
f\left(x y^{-1}\right)+f\left(x y^{2}\right)=f(x y)-3 f(x),
$$

which yields the desired result when combined with Lemma 5.

Lemma 8 Let $f \in \mathscr{A}\left(G, G^{*}\right)$ and let $x, y \in G$. If

$$
\begin{array}{r}
f\left(x y^{-1}\right)+f(x y)=-2 f(x)-2 f(y), \\
f(x)+f\left(x y^{-2}\right)=2 f(x y)+2 f(y),
\end{array}
$$

then $f(x)= \pm f(y)$ or $f\left(x y^{-1}\right)=f(x) \pm 2 f(y)$.

Proof: Replacing $y$ in Lemma 7 with its inverse and using $f\left(y^{-1}\right)=f(y)$ from Theorem 1 , we get the desired result.

The following theorem gives a partial result towards the equivalence of the alternative quadratic functional equation (3) and the quadratic functional equation (4) when the function values at some points vanish.

Theorem 4 Let $f \in \mathscr{A}\left(G, G^{*}\right)$ and let $x, y \in G$. If $f(y)=0$, then $f\left(x y^{-1}\right)+f(x y)=2 f(x)$.

Proof: Let $f \in \mathscr{A}\left(G, G^{*}\right)$. Suppose there exist $x, y \in$ $G$ such that $f(y)=0$ but

$$
f\left(x y^{-1}\right)+f(x y) \neq 2 f(x) .
$$

From $\mathscr{P}_{f}(x, y)$, we will have

$$
f\left(x y^{-1}\right)+f(x y)=-2 f(x) .
$$

Observe from (24) and (25) that $f(x) \neq 0$. Consider the two possibilities on $\mathscr{P}_{f}(x y, y)$.

We first consider the case when

$$
f(x)+f\left(x y^{2}\right)=2 f(x y) .
$$

Applying Lemma 7 to (25) and (26), then noting that $f(y)=0$ and $f(x) \neq 0$, we are left with $f(x y)=f(x)$. As a result, (25) gives $f\left(x y^{-1}\right)=$ $-3 f(x)$. Considering $\mathscr{P}_{f}\left(x y^{-1}, y\right)$, we will have

$$
f\left(x y^{-2}\right) \in\{5 f(x),-7 f(x)\} .
$$

On the other hand, replacing $x$ in Lemma 5 with $x y^{-1}$, we derive

$$
f\left(x y^{-2}\right) \in\{f(x),-3 f(x)\} .
$$

Comparing (27) and (28), we infer that $f(x)=0$, a contradiction.
Now we turn to the case when

$$
f(x)+f\left(x y^{2}\right)=-2 f(x y) .
$$

Applying Lemma 6 to (25) and (29), then realizing that $f(y)=0$, we get

$$
f(x)+f(x y)=0 .
$$

Since $(G, \cdot)$ is 2-divisible, there exists $z \in G$ such that $y=z^{2}$. Therefore $\mathscr{P}_{f}(x z, z)$ and (30) give $f(x z)=$ 0 . Eliminating $f(x y)$ from (25) and (30) gives

$$
f\left(x y^{-1}\right)+f(x)=0 .
$$

Then $\mathscr{P}_{f}\left(x z^{-1}, z\right)$ and (31) yields $f\left(x z^{-1}\right)=0$. Finally, $\mathscr{P}_{f}(x, z)$ with $f\left(x z^{-1}\right)+f(x z)=0$ gives $f(x)=0$, a contradiction.

\section{MAIN RESULTS}

In this section, we shall use the lemmas and the theorems in the previous sections to obtain the equivalence of (3) and (4) by settling each combination of alternatives in $\mathscr{P}_{f}\left(x y^{-1}, y\right)$ and $\mathscr{P}_{f}(x y, y)$.

Theorem $5 f \in \mathscr{A}\left(G, G^{*}\right)$ if and only if $f$ satisfies the quadratic functional equation (4) for all $x, y \in G$.

Proof: The sufficiency of the theorem is obvious. For the necessity, let $f \in \mathscr{A}\left(G, G^{*}\right)$. Suppose there exist $x, y \in G$ such that

$$
f\left(x y^{-1}\right)+f(x y) \neq 2 f(x)+2 f(y) .
$$

We will denote $\alpha=f(y)$ and $a_{n}=f\left(x y^{n}\right)$ for every $n \in \mathbb{Z}$. Therefore (32) becomes

$$
a_{-1}+a_{1} \neq 2 a_{0}+2 \alpha .
$$

From $\mathscr{P}_{f}(x, y)$ and (33), we have

$$
a_{-1}+a_{1}=-2 a_{0}-2 \alpha .
$$

From (33) and (34), we observe that

$$
a_{-1}+a_{1} \neq 0 .
$$

In addition, (33) and the contrapositive of Theorem 4 imply that

$$
\alpha \neq 0 \text {. }
$$

Considering $\mathscr{P}_{f}\left(x y^{-1}, y\right)$ and $\mathscr{P}_{f}(x y, y)$, there exist $\varepsilon_{1}, \varepsilon_{2} \in\{-1,1\}$ such that

$$
\begin{aligned}
a_{-2}+a_{0} & =2 \varepsilon_{1}\left(a_{-1}+\alpha\right), \\
a_{0}+a_{2} & =2 \varepsilon_{2}\left(a_{1}+\alpha\right) .
\end{aligned}
$$


Considering $\mathscr{P}_{f}\left(x, y^{2}\right)$ and using $f\left(y^{2}\right)=4 f(y)=$ $4 \alpha$, we have

$$
a_{-2}+a_{2}= \pm 2\left(a_{0}+4 \alpha\right) .
$$

Combining (37), (38) and (39), we have

$$
\begin{gathered}
\varepsilon_{1}\left(a_{-1}+\alpha\right)+\varepsilon_{2}\left(a_{1}+\alpha\right) \in\left\{2 a_{0}+4 \alpha,-4 \alpha\right\} \\
\text { If } \varepsilon_{1}=\varepsilon_{2}=1 \text {, then (33) and (40) give } \\
a_{-1}+a_{1}=-6 \alpha .
\end{gathered}
$$

Then (34) gives $a_{0}=2 \alpha$. Observing (36) and $a_{0} \neq$ $\pm \alpha$, we apply Lemma 8 to (37) and (34) to get

$$
a_{-1} \in\{0,4 \alpha\} .
$$

Apply Lemma 7 to (34) and (38), while observing that $a_{0} \neq \pm \alpha$,

$$
a_{1} \in\{0,4 \alpha\} .
$$

From (41), (42) and (43), we infer that $\alpha=0$, a contradiction.

$$
\text { If } \varepsilon_{1}=1 \text { and } \varepsilon_{2}=-1 \text {, then (40) becomes }
$$$$
a_{-1}-a_{1} \in\left\{-4 \alpha, 2 a_{0}+4 \alpha\right\} .
$$

Eliminating $a_{-1}$ from (34) and (44), we have

$$
a_{0}+a_{1}=\alpha \quad \text { or } 2 a_{0}+a_{1}=-3 \alpha .
$$

Apply Lemma 6 to (34) and (38) to get

$$
a_{0}+a_{1} \in\{0,-2 \alpha,-4 \alpha\} .
$$

Combining (45) and (46), there remain three possibilities:

(a) $a_{0}=-3 \alpha$ and $a_{1}=3 \alpha$. From (34), we have $a_{-1}=\alpha$. From (37), we have $a_{-2}=7 \alpha$. Considering Lemma 5 with $x$ replaced by $x y^{-1}$, we conclude that $\alpha=0$, a contradiction.

(b) $a_{0}=a_{1}=-\alpha$. From (34), we have $a_{-1}+a_{1}=0$, which contradicts (35).

(c) $a_{0}=\alpha$ and $a_{1}=-5 \alpha$. From (34), we have $a_{-1}=\alpha$. From (38), we have $a_{2}=7 \alpha$. Considering $\mathscr{P}_{f}\left(x y^{2}, y\right)$, we have $a_{3} \in\{21 \alpha,-11 \alpha\}$. On the other hand, $\mathscr{P}_{f}\left(x y, y^{2}\right)$ gives $a_{3} \in$ $\{\alpha,-3 \alpha\}$. Comparing the possible values of $a_{3}$, we infer that $\alpha=0$, a contradiction.

If $\varepsilon_{1}=-1$ and $\varepsilon_{2}=1$, then we switch the roles of $a_{n}$ and $a_{-n}$, for every $n \in \mathbb{N}$, in the argument when $\varepsilon_{1}=1$ and $\varepsilon_{2}=-1$. This will lead us to the same contradictions.
If $\varepsilon_{1}=\varepsilon_{2}=-1$, then (34) and (40) give $a_{0}=$ $-2 \alpha$ and

$$
a_{-1}+a_{1}=2 \alpha .
$$

Apply Lemma 6 with $x y^{-1}$ instead of $x$ to (37) and (34) to get

$$
a_{-1} \in\{2 \alpha, 0,-2 \alpha\} .
$$

Apply Lemma 6 again to (34) and (38) to get

$$
a_{1} \in\{2 \alpha, 0,-2 \alpha\} .
$$

From (47), (48) and (49), there are two possible cases:

(a) $a_{-1}=0$ and $a_{1}=2 \alpha$. From (37), we get $a_{-2}=$ 0 . Considering $\mathscr{P}_{f}\left(x y^{-2}, y\right)$, we have $a_{-3}=$ $\pm 2 \alpha$. On the other hand, $\mathscr{P}_{f}\left(x y^{-1}, y^{2}\right)$ gives $a_{-3} \in\{6 \alpha,-10 \alpha\}$. Comparing $a_{-3}$, we infer that $\alpha=0$, a contradiction.

(b) $a_{-1}=2 \alpha$ and $a_{1}=0$. Switching the roles of $a_{n}$ and $a_{-n}$, for every $n \in \mathbb{N}$, in the argument of the case (a) will lead us to the same contradiction.

\section{REFERENCES}

1. Kuczma M (1978) On some alternative functional equations. Aequat Math 17, 182-98.

2. Kuczma M (1978) Functional equations on restricted domains. Aequat Math 18, 1-34.

3. Forti GL (1979) La soluzione generale dell'equazione funzionale $\{c f(x+y)-a f(x)-b f(y)-d\}\{f(x+y)-$ $f(x)-f(y)\}=0$. Matematiche 34, 219-42.

4. Ger R (1976) On a method of solving of conditional Cauchy equations. Univ Beograd Publ Elektrotehn Fak Ser Mat Fiz 544-576, 159-65.

5. Forti GL, Paganoni L (1981) A method of solving of a conditional Cauchy equation on abelian groups. Ann Mat Pura Appl 127, 77-99.

6. Paganoni L, Rätz J (1995) Conditional functional equation and orthogonal additivity. Aequat Math 50, 135-42.

7. Ger R (1993) On a characterization of strictly convex spaces. Atti Accad Sci Torino Cl Sci Fis Mat Natur 127, 131-8.

8. Ger R (2014) Fischer-Muszély functional equation almost everywhere. Aequat Math 89, 207-14.

9. Skof F (1995) On some alternative quadratic equations. Results Math 27, 402-11. 